\title{
Detection of $\mathrm{HCO}^{+}$emission toward the PN K 3-35
}

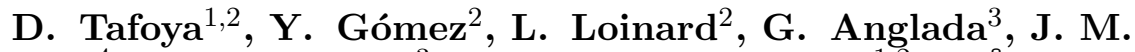 \\ Torrelles $^{4}$, L. F. Miranda ${ }^{3}$, R. Franco-Hernández ${ }^{1,2}$, L-Å. Nyman ${ }^{5}$, \\ and J. Nakashima ${ }^{6}$ \\ ${ }^{1}$ Harvard-Smithsonian Center for Astrophysics, 60 Garden Street, \\ Cambridge, MA 02138, USA \\ ${ }^{2}$ Centro de Radioastronomía y Astrofísica, UNAM Apartado Postal \\ 3-72 (Xangari), 58089 Morelia, Michoacán, México. \\ ${ }^{3}$ Instituto de Astrofísica de Andalucía, CSIC Apartado 3004, E-18080, Granada, Spain. \\ ${ }^{4}$ Instituto de Ciencias del Espacio, Consejo Superior de Investigaciones Científicas-Instit \\ D'Estudis Espacials de Catalunya, (CSIC-IEEC), Gran Capità 2, E-08034 Barcelona, Spain. \\ ${ }^{5}$ Swedish-ESO Submillimetre Telescope, European Southern Observatory, Casilla 19001, \\ Santiago 19, Chile; Onsala Space Observatory, 43992 Onsala Sweden. \\ ${ }^{6}$ Institute of Astronomy and Astrophysics, Academia Sinica, Taipei, Taiwan
}

\begin{abstract}
Here we are reporting the detection of $\mathrm{HCO}^{+}(J=1 \rightarrow 0)$ emission as well as weak emission of $\mathrm{CO}(J=1 \rightarrow 0)$ toward the planetary nebula $(\mathrm{PN}) \mathrm{K} 3-35$ as a result of a molecular emission survey carried out toward this source. K 3-35 is remarkable because it is one of the two $\mathrm{PNe}$ that are known to exhibit water maser emission. In this nebula, the emission is present in the central region as well as at a distance of $\simeq 5000 \mathrm{AU}$ away from the center. The presence of molecular emission reveals some clues that could lead to the understanding of the persistence of water molecules in its envelope. We also report new spectra of the CO $(J=2 \rightarrow 1)$ transition. From the $\mathrm{CO}$ emission we have obtained a value for the excitation temperature of the molecular gas of $\simeq 20 \mathrm{~K}$. Using this result, we have estimated a molecular mass for the envelope of $\simeq 0.017 M_{\odot}$, and that the abundance for the $\mathrm{HCO}^{+}$is $6.1 \times 10^{-7}$.
\end{abstract}

Keywords. Planetary Nebula — Stars: individual (K 3-35) - Radio lines: molecules — Stars: circumstellar matter

\section{Introduction}

$\mathrm{K} 3-35$ is one of the two planetary nebulae $(\mathrm{PNe})$ that have been found to harbor water maser emission (Miranda et al. 2001). Water-vapor masers that are detected in the envelopes of red giants (Elitzur 1992; Habing 1996) and also in some proto-PNe (Likkel \& Morris 1988; Imai et al. 2002), are not expected to persist in the PN phase where the envelope begins to be ionized (Gómez et al. 1990). Therefore, the presence and persistence of water molecules in this PN is puzzling, probably related to some shielding mechanism due to the presence of high density molecular gas that protects water molecules against the ionizing radiation of the central star. A full understanding of this shielding mechanism requires first the characterization of the physical conditions of the molecular gas around the planetary nebula together with detailed modeling studies. Any information about the chemistry and physical parameters of the gas in K3-35 is very important to understand the characteristics of such a particular object. In order to find some clues that might help to better understand this object, we carried out a survey for molecular emission having as a result the first detection of the $\mathrm{HCO}^{+}(J=1 \rightarrow 0)$ line emission toward this young planetary nebula. 


\section{Observations and Results}

The survey for molecular lines toward the young planetary nebula K 3-35 was carried out using the Onsala $20 \mathrm{~m}$ telescope in Sweden (OSO), the $45 \mathrm{~m}$ Nobeyama telescope in Japan and the IRAM $30 \mathrm{~m}$ telescope in Spain and included the following molecules: $\mathrm{SiO}, \mathrm{HCO}^{+}, \mathrm{H}^{13} \mathrm{CO}^{+}, \mathrm{HNC}, \mathrm{HCN}, \mathrm{HC}_{5} \mathrm{~N}, \mathrm{HC}_{3} \mathrm{~N}, \mathrm{CS}, \mathrm{CN}, \mathrm{CH}_{3} \mathrm{OH}, \mathrm{CO}$ and ${ }^{13} \mathrm{CO}$. We succeeded in detecting for the first time weak emission of $\mathrm{HCO}^{+}(J=1 \rightarrow 1)$ with the $20 \mathrm{~m}$ Onsala telescope $\left(\mathrm{HPBW}=44^{\prime \prime}\right)$. Figure 1 a shows the continuum subtracted spectrum in main beam brightness temperature. $\operatorname{CO}(J=1 \rightarrow 0)$ emission also was detected for the first time using the Nobeyama $45 \mathrm{~m}$ telescope $\left(\mathrm{HPBW}=16^{\prime \prime}\right.$, lower panel of Figure $\left.1 \mathrm{~b}\right)$. Finally, we detected CO $(J=2 \rightarrow 1)$ emission toward K $3-35$ using the IRAM $30 \mathrm{~m}$ telescope (HPBW=12", upper panel of Figure $1 \mathrm{~b})$.
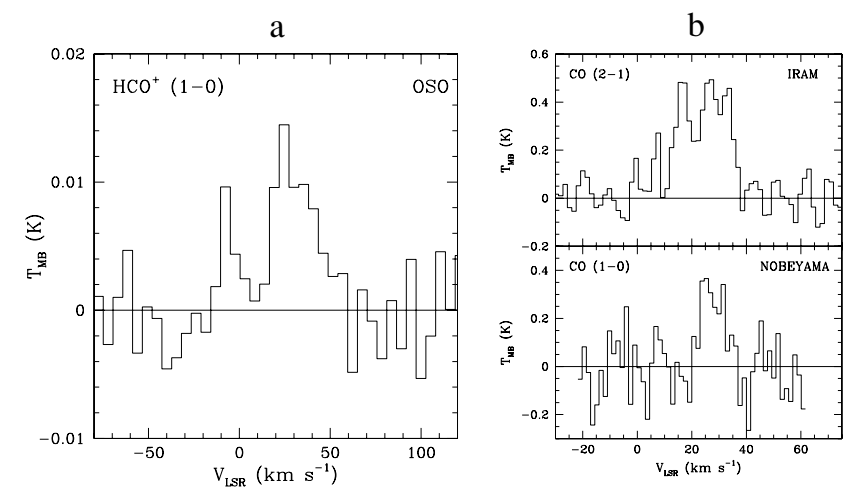

Figure 1. Left: Onsala telescope $\mathrm{HCO}^{+}(J=1 \rightarrow 0)$ spectrum. Right (upper): $\mathrm{CO}(J=2 \rightarrow 1)$ spectra obtained with the IRAM $30 \mathrm{~m}$ telescope. Rigth (Lower):CO $(J=1 \rightarrow 0)$ spectra obtained with the Nobeyama $45 \mathrm{~m}$ telescope.

In order to determine the physical parameters of the K 3-35 envelope it is necessary to determine the excitation temperature $\left(T_{\mathrm{ex}}\right)$. In trying to improve previous results, we used our CO observations and derived a value of $20 \pm 6 \mathrm{~K}$ for this temperature. For this value of $T_{\text {ex }}$, we estimated that the column densities of $\mathrm{CO}$ and $\mathrm{HCO}^{+}$, averaged over their respective beams, are: $N(\mathrm{CO})=4.95 \times 10^{15} \mathrm{~cm}^{-2}$ and $N\left(\mathrm{HCO}^{+}\right) \simeq 6.95 \times$ $10^{11} \mathrm{~cm}^{-2}$. This results in a total molecular mass for the $\mathrm{K} 3-35$ evelope of $M_{\mathrm{mol}} \simeq$ $0.017 M_{\odot}$, and an abundace for the $\mathrm{HCO}^{+}$of $X_{\mathrm{HCO}^{+}}=6.1 \times 10^{-7}$.

\section{References}

Dayal, A. \& Bieging, J. H. 1996, ApJ, 472, 703

de Gregorio-Monsalvo, I., Gómez, Y., Anglada, G., Cesaroni, R., Miranda, L. F., Gómez, J. F. \& Torrelles, J. M. 2004, ApJ, 601, 921

Elitzur, M. 1992, ARA\&A, 30, 75

Gómez, Y., Moran, J. M. \& Rodríguez, L. F. 1990, RevMexAA, 20, 55

Habing, H. J. 1996, A\&ARv, 7, 97

Huggins, P. J., Bachiller, R., Planesas, P., Forveille, T. \& Cox, P. 2005, ApJS 160272

Imai, H., Obara, K., Diamond, P. J., Omodaka, T. \& Sasao, T. 2002, Nature, 417, 829

Likkel, L. \& Morris, M. 1988, ApJ, 329, 914

Miranda, L. F., Gómez, Y., Anglada, G. \& Torrelles, J. M. 2001, Nature, 414, 28 